\title{
Focused Ion Beam preparation and characterization of single-crystal samples for high-pressure experiments in the diamond-anvil cell
}

\author{
Hauke Marquardt $^{1}$, Katharina Marquardt ${ }^{1}$, Sergio Speziale ${ }^{1}$ \\ ${ }^{1}$ Deutsches GeoForschungsZentrum, Section 3.3, Potsdam, Germany
}

High-pressure experiments in the diamond-anvil cell (DAC) give important insights into the properties of materials at extreme pressure and temperature conditions. The available space in the pressure chamber of a DAC, however, is very limited. It is often challenging to mechanically prepare singlecrystal samples with suitable dimensions for experiments in the DAC, particularly at extreme pressure conditions. In addition, a number of experimental techniques, such as Brillouin spectroscopy, require well-polished sample surfaces. Mechanical polishing, however, is restricted to materials of sufficient size and mechanical stability. This precludes the preparation of a number of compounds with significant geophysical relevance, including both natural samples (such as serpentine minerals) and candidate Earth materials that are synthesised at high-pressure/high-temperature conditions (such as ferropericlase or $\mathrm{MgSiO}_{3}$-perovskite).

In this contribution, we discuss the use of focused ion beam (FIB) devices to prepare single-crystal samples with dimensions of few tens of microns and precisely defined shapes that can be probed by several techniques at extreme conditions. The procedure discussed here does not expose the samples to mechanical forces and thus allows for preparing materials that are brittle, meta-stable, or show a strong cleavage. In addition, it allows us to cut more than one sample from a piece of material, for instance platelets with different orientations from one single-crystal. Also, an electron transparent foil can be produced simultaneously. This allows for a detailed TEM-characterization - including chemical composition, crystallographic orientation, defect structure and secondary phases - of the same sample material, i.e. the same single-crystal, that is later used in the high-pressure experiment. We demonstrate the capabilities of this approach using several different geomaterials, including perovskite (fig. 1), ferropericlase, spinel, and antigorite. We will focus on the suitability of the prepared samples for Brillouin spectroscopy.

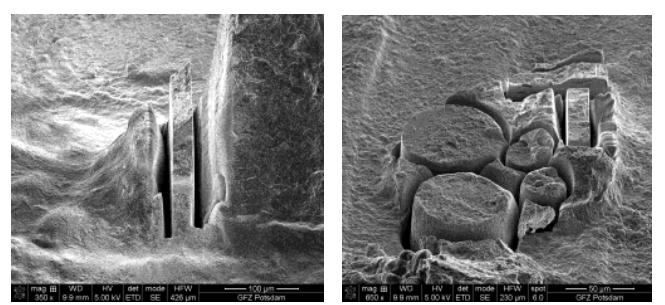

Figure 1: Secondary electron images of a perovskite single-crystal platelet that was cut from an aggregate made up of several single-crystals. The platelet, with approximate dimensions of $180 \times 180$ x $30 \mu \mathrm{m}^{3}$, was double-side polished (left). The platelet was manually flipped by $90^{\circ}$ and again inserted into the dual beam machine (right). Several samples were cut for Brillouin spectroscopy, single-crystal $\mathrm{x}$-ray diffraction and TEM analysis. 